\title{
Sleep apnoea is associated with major cardiac events in peripheral arterial disease
}

\author{
Karri T. Utriainen ${ }^{1,2}$, Juhani K. Airaksinen ${ }^{3}$, Olli Polo ${ }^{2,4}$, Ruut Laitio ${ }^{5}$, \\ Mikko J. Pietilä ${ }^{3}$, Harry Scheinin ${ }^{5}$, Tero Vahlberg 6 , Kari A. Leino ${ }^{5}$, \\ Erkki S. Kentala ${ }^{5}$, Jouko R. Jalonen ${ }^{5}$, Harri Hakovirta7, Riitta Parkkola ${ }^{8}$, \\ Sami Virtanen ${ }^{8}$ and Timo T. Laitio ${ }^{5}$
}

Affiliations: ${ }^{1}$ Division of Medicine, Turku University Hospital, Turku, ${ }^{2}$ Sleep Research Unit, University of Turku, Turku, ${ }^{3}$ Heart Centre, Turku University Hospital and University of Turku, Turku, ${ }^{4}$ Dept of Pulmonology, Tampere University Hospital, Tampere, ${ }^{5}$ Division of Perioperative Services, Intensive Care Medicine and Pain Management, Turku University Hospital, Turku, ${ }^{6}$ Dept of Biostatistics, University of Turku, Turku, ${ }^{7}$ Dept of Vascular Surgery, Turku University Hospital, Turku, and ${ }^{8}$ Dept of Radiology, Turku University Hospital, Turku, Finland.

Correspondence: T.T. Laitio, Division of Perioperative Services, Intensive Care Medicine and Pain Management, P.O.B. 52, FIN-20521, Turku, Finland. E-mail: timo.laitiolaelisanet.fi

ABSTRACT Obstructive sleep apnoea (OSA) is associated with atherosclerosis and cardiovascular events. Peripheral arterial disease (PAD) represents severe atherosclerosis with a high mortality after vascular surgery. The role of OSA in the prognosis of these patients is not yet established.

84 patients (aged $67 \pm 9$ years) scheduled for sub-inguinal surgical revascularisation were enrolled for preoperative polysomnography. The threshold for significant OSA was an apnoea/hypopnoea index $\geqslant 20$ events $\cdot h^{-1}$. Major adverse cardiovascular and cerebrovascular events (MACCE), including cardiac death, myocardial infarction, coronary revascularisation, angina pectoris requiring hospitalisation and stroke, were used as a combined end-point.

During follow-up (median 52 months), 17 out of 39 patients with and six out of 45 patients without significant OSA suffered MACCE. In the multivariate Cox regression, the primary predictors of MACCE were significant OSA (hazard ratio (HR) $5.1(95 \%$ CI 1.9-13.9); $\mathrm{p}=0.001)$ and pre-existing coronary artery disease (HR 4.4 (95\% CI 1.8-10.6); $\mathrm{p}=0.001)$. Other significant predictors were a $\geqslant 4$ year history of PAD (HR 3.8 (95\% CI 1.3-11.5); $\mathrm{p}=0.02$ ) and decreasing high-density lipoprotein/total cholesterol ratio (HR 0.95 per percentage ( $95 \%$ CI $0.90-1.00) ; \mathrm{p}=0.048$ ).

OSA is associated with poor long-term outcome in patients with PAD following revascularisation. OSA might have an important role in the pathogenesis of cardiovascular morbidity and mortality in these patients.

@ERSpublications

Sleep apnoea is associated with poor long-term outcome in peripheral arterial disease patients undergoing surgery http://ow.ly/sL8T0

Received: July 292013 | Accepted after revision: Dec 292013 | First published online: Feb 202014

Clinical trial: This study is registered at www.ClinicalTrials.gov (identifier NCT00712946).

Support statement: This work was supported by GE Healthcare Finland (Helsinki, Finland), the Finnish Funding Agency for Technology and Innovation (TEKES; Helsinki, Finland), the Clinical Research Fund (EVO) of Turku University Hospital (Turku, Finland) and the Instrumentarium Science Foundation (Helsinki, Finland).

Conflict of interest: Disclosures can be found alongside the online version of this article at www.erj.ersjournals.com

Copyright @ERS 2014 


\section{Introduction}

Peripheral arterial disease (PAD) represents a severe form of systemic atherosclerosis with a high cardiovascular morbidity regardless of clinical manifestations in other vascular beds [1]. Patients with PAD undergoing sub-inguinal surgical revascularisation have a poor prognosis with a 5-year mortality of $\sim 30 \%$, which is mostly caused by cardiac complications [2]. Because these events represent a major clinical problem in terms of increased morbidity and healthcare expenses, preoperative noninvasive cardiac assessment and invasive coronary revascularisation for high-risk patients is recommended to improve longterm outcome [3]. However, the underlying pathophysiological mechanisms and prognostic factors are not yet established and studies aiming to identify which patients are at the greatest risk have yielded controversial or disappointing results $[4,5]$.

Obstructive sleep apnoea (OSA) is common in patients with cardiovascular disease [6, 7]. Increasing evidence suggests that OSA is associated with the risk factors, development and progression of atherosclerosis. Earlier studies have shown that OSA is independently related to both hypertension and diabetes [8,9]. Furthermore, it has been demonstrated that OSA, even when minimally symptomatic, promotes oxidative stress, systemic inflammation, endothelial dysfunction and arterial stiffness, the key factors in the pathogenesis of atherosclerosis $[10,11]$. It has been stated that OSA combined with other risk factors has an incremental role in this pathophysiological cascade [12]. Finally, OSA is associated with the progression of atherosclerotic disease along with both fatal and non-fatal cardiovascular events [13-16].

We have previously shown that OSA (apnoea/hypopnoea index (AHI) $\geqslant 5$ events $\cdot \mathrm{h}^{-1}$ ) is exceedingly common in PAD patients undergoing surgical peripheral revascularisation and it is mostly unrecognised [17]. The purpose of this study is to evaluate the unestablished association of this occult OSA with longterm cardiac morbidity and mortality in PAD patients by assessing our previously described population after 1-7 years of follow-up [17].

\section{Materials and methods}

\section{Study subjects}

This study is part of the BAROSLEEP trial (www.ClinicalTrials.gov identifier NCT00712946), which is designed to increase our understanding of the pathophysiology responsible for the poor long-term outcome of PAD patients. In this study, the impact of OSA was determined on long-term cardiovascular morbidity and mortality in the same PAD patients who were previously shown to have highly prevalent OSA [17]. Patients aged $>40$ years with PAD referred to Turku University Hospital (Turku, Finland) for elective sub-inguinal revascularisation were eligible for this prospective study. Exclusion criteria were pre-existing OSA syndrome, congestive heart failure, atrial fibrillation, inability to cooperate, end-stage renal disease, coronary bypass within 3 years or other major surgery within 3 months prior to enrolment. The recruitment was carried out between April 2006 and December 2011. The study was approved by the Ethics Committee of the Hospital District of Southwest Finland (Turku, Finland).

\section{Data collection and analysis}

All patients underwent a detailed clinical evaluation, including preoperative echocardiography. Lipid profile, including high-density lipoprotein (HDL)/total cholesterol ratio was determined from fasting blood samples or collected from hospital records. A glucose tolerance test was performed in patients without preexisting diabetes [18]. Coronary artery disease and hypertension were considered present if previously diagnosed. Metabolic syndrome was diagnosed according to the latest recommendations [19].

All subjects underwent an overnight polysomnography (Embla/Somnologica; MedCare, Reykjavik, Iceland) as previously described $[17,20]$. The recordings were analysed according to the recommended guidelines [21]. Respiration was monitored with a pressure transducer attached to nasal prongs for respiratory flow. A pulse oximeter was utilised to measure arterial oxyhaemoglobin saturation and plethysmography. Apnoea was defined as cessation of airflow for at least $10 \mathrm{~s}$. Hypopnoea was defined as a discernible tidal volume reduction of $>50 \%$, associated with $\geqslant 4 \%$ oxygen desaturation [20]. The AHI was calculated as the number of these respiratory events per hour of sleep. Arterial oxygen desaturation index (ODI) was determined as the number of desaturations of at least $4 \%$ per hour of sleep, and was used instead of the AHI in four patients in whom the nasal prongs had been detached during the recording. Central and obstructive apnoea episodes were identified according to the respiratory swing observed in pulse transit time, calculated from the electrocardiogram and plethysmography signals [22, 23]. The Epworth Sleepiness Scale and the Berlin Questionnaire were used to assess symptoms of sleep apnoea [24, 25].

Cardiac troponin $\mathrm{T}$ was measured on the first three post-operative days. All patients were routinely seen at the outpatient ward 6 weeks and 1 year post-operatively. Following this, they were contacted annually by telephone, and major adverse events were retrieved from hospital records. Long-term follow-up was 
continued until February 2013. The combined end-point of major adverse cardiovascular and cerebrovascular events (MACCE) was defined as cardiac death, acute myocardial infarction (AMI), coronary revascularisation, unstable angina pectoris needing hospitalisation and stroke. AMI was determined according to the third universal definition [26].

\section{Statistical analysis}

To determine the risk of MACCE related to OSA, preliminary analyses were performed with increments of 10 events $\cdot h^{-1}$ in the AHI, i.e. the study sample was divided into patients with AHI $0-10$ events $\cdot h^{-1}$, 10-20 events $\cdot \mathrm{h}^{-1}, 20-30$ events $\cdot \mathrm{h}^{-1}$ and $>30$ events $\cdot \mathrm{h}^{-1}$; the latter three groups were compared to the first. According to these analyses and previous literature, AHI $\geqslant 20$ events $\cdot \mathrm{h}^{-1}$ was finally used as a threshold for significant OSA [16, 27]. The differences between patients with AHI $<20$ events $\cdot \mathrm{h}^{-1}$ and AHI $\geqslant 20$ events $\cdot \mathrm{h}^{-1}$ were tested using the Mann-Whitney U-test for continuous variables and Fisher's exact test for categorical variables. The Shapiro-Wilk test was used to test for normal distribution of data. Univariate Cox regression analysis was used first to determine variables associated with MACCE. All clinical variables recorded at baseline were tested separately (i.e. age, sex, smoking status, PAD history, history of stroke, presence of coronary artery disease, metabolic syndrome, diabetes, hypertension, critical limb ischaemia, ankle-brachial index, body mass index, waist circumference, left ventricular ejection fraction, lipid parameters). Of the sleep variables, significant OSA (AHI $\geqslant 20$ events $\left.\cdot h^{-1}\right)$, central sleep apnoea, arousal index, mean and lowest oxygen saturation (arterial oxygen saturation $\left(\mathrm{SaO}_{2}\right)$ mean and nadir), time below 90\% saturation $\left(\mathrm{SaO}_{2}\right.$ T90), Epworth Sleepiness Scale score and pathological Berlin Questionnaire results (at least two positive categories) were tested. In these analyses, the duration of PAD history was used as a categorical variable ( $0-3$ years and $\geqslant 4$ years). Variables significantly associated with the occurrence of MACCE in the univariate analysis were included in a multivariate Cox regression analysis using a stepwise selection method (inclusion criteria $\mathrm{p}<0.05$ and exclusion criteria $\mathrm{p} \geqslant 0.05$ ). The results are presented using hazard ratio (HR) with 95\% confidence intervals. A Kaplan-Meier analysis was used to show the impact of OSA on MACCE-free survival. Two-tailed p-values $<0.05$ were considered statistically significant. The statistical analyses were performed with SAS 9.2 software for Windows (SAS Institute Inc., Cary, NC, USA).

\section{Results}

\section{Patient characteristics}

A total of 145 patients fulfilling the inclusion criteria and none of the exclusion criteria were eligible for the study. Of these, 59 patients refused to participate and 86 patients gave their written informed consent. After rejection of two patients because of insufficient sleep data due to technical problems, 84 patients were included in the final analyses, i.e. the same patients as in our previous study [17]. A flow chart detailing the number of excluded patients for each exclusion criterion is shown in figure 1. Demographic features and clinical data of the enrolled patients are summarised in table 1. All of the included subjects were independently dwelling outpatients. Eligible patients who refused consent were significantly older (71 versus 67 years, $p=0.02$ ) but did not differ in any of the available clinical parameters (data not shown). Left ventricular ejection fraction was mildly decreased (40-49\%) in four patients with AHI $\geqslant 20$ events $\cdot \mathrm{h}^{-1}$; the other patients had normal systolic function (table 1). The patients' long-term medication use is detailed in table 2.

Sleep apnoea was diagnosed in the majority of patients, as reported previously [17]. For this study, significant OSA with an AHI $\geqslant 20$ events $\cdot \mathrm{h}^{-1}$ was observed in $39(46 \%)$ patients (95\% CI 36-57\%). ODI (mean 23 events $\cdot \mathrm{h}^{-1}$, median (interquartile range) $17(8-34)$ events $\cdot \mathrm{h}^{-1}$ ) and AHI (mean 23 events $\cdot \mathrm{h}^{-1}$, median (interquartile range) $18(9-35)$ events $^{-1} \mathrm{~h}^{-1}$ ) had a high correlation in the whole study population (Spearman's coefficient $\mathrm{r}=0.92$ ). The episodes of apnoea were predominantly obstructive. OSA was unrelated to obesity and was asymptomatic, as measured with the Epworth Sleepiness Scale and the Berlin Questionnaire. Sleep variables are described in table 3.

\section{Follow-up and major end-points}

After a median follow-up of 52 months (range 14-84 months), 18 patients had died. Of these, nine deaths were due to cardiac events, two due to cerebrovascular events, and seven due to other causes (cancer, septicaemia, liver cirrhosis, pulmonary fibrosis and alcoholism). Altogether, MACCE was observed in 23 patients (table 4), and they occurred within a median follow-up of 16 months (range 0-75 months). Of the 23 MACCE, one AMI and one cardiac death occurred within 30 days, and one perioperative myocardial infarction (elevated cardiac troponin T) within 3 days after surgery. None of the medications used by the patients had a significant effect on outcome. 


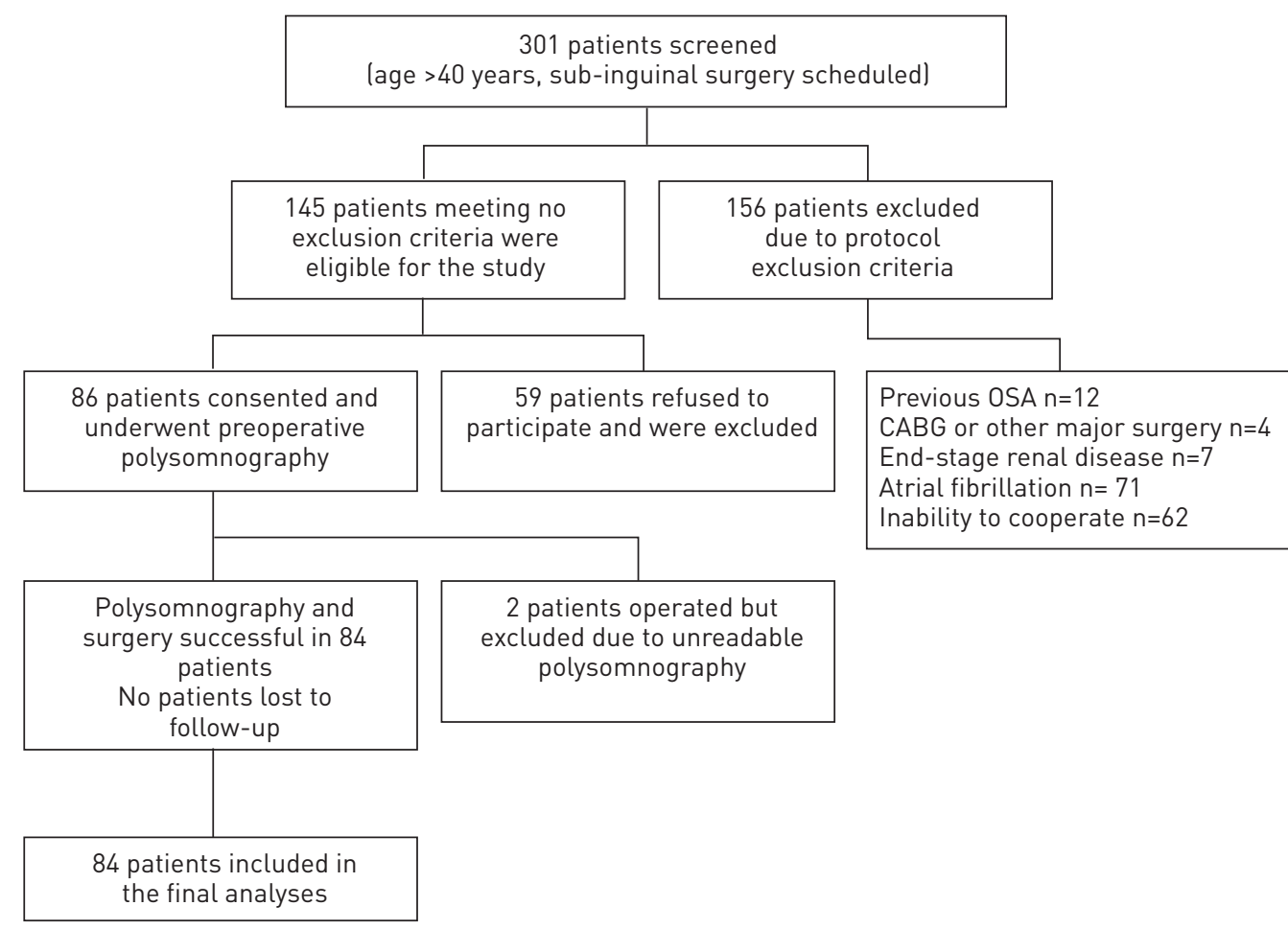

FIGURE 1 Flow chart of study patients from screening to end-point analysis. OSA: obstructive sleep apnoea; CABG: coronary artery bypass grafting.

\section{Predictors of MACCE}

In the preliminary analyses with different AHI cut-off levels (compared to patients with AHI 0-10 events $\cdot \mathrm{h}^{-1}$ ), an AHI 20-30 events $\cdot \mathrm{h}^{-1}$ (HR 3.0 (95\% CI 0.9-10.5); $\left.\mathrm{p}=0.08\right)$ and an AHI >30 events $\cdot \mathrm{h}^{-1}$ (HR 3.4 (95\% CI 1.0-11.3); $\mathrm{p}=0.04)$ turned out to be the most likely predictors of MACCE. OSA with an AHI

\section{TABLE 1 Baseline characteristics according to severity of obstructive sleep apnoea}

\begin{tabular}{|c|c|c|c|}
\hline & All patients & $\mathrm{AHI}^{\#}<20$ events $\cdot \mathrm{h}^{-1}$ & $A H I^{\#} \geqslant 20$ events $\cdot h^{-1}$ \\
\hline Subjects & 84 & 45 & 39 \\
\hline Age years & $67 \pm 9$ & $65 \pm 8$ & $70 \pm 8^{*}$ \\
\hline Male & $52(62)$ & $24(53)$ & $28(72)$ \\
\hline Smoker & 32 (38) & $20(44)$ & $12(31)$ \\
\hline Metabolic syndrome & $51(61)$ & $24(55)$ & 27 (69) \\
\hline $\mathrm{BMI} \mathrm{kg} \cdot \mathrm{m}^{-2}$ & $26(24-29)$ & $26(24-29)$ & $27(24-29)$ \\
\hline Diabetes & $36(43)$ & $21(47)$ & 15 (39) \\
\hline Arterial hypertension & $70(83)$ & $35(78)$ & $35(90)$ \\
\hline Coronary artery disease & $31(37)$ & $15(33)$ & $16(41)$ \\
\hline Stroke & $14(17)$ & $7(16)$ & $7(18)$ \\
\hline LVEF \% & $63 \pm 8$ & $67 \pm 6$ & $59 \pm 8^{\circ}$ \\
\hline Cholesterol $\mathrm{mmol} \cdot \mathrm{L}^{-1}$ & $4.3 \pm 1.0$ & $4.2 \pm 1.0$ & $4.5 \pm 1.1$ \\
\hline LDL $\mathrm{mmol} \cdot \mathrm{L}^{-1}$ & $2.2 \pm 0.8$ & $2.1 \pm 0.8$ & $2.3 \pm 0.8$ \\
\hline HDL/cholesterol \% & $34 \pm 10$ & $36 \pm 10$ & $32 \pm 9$ \\
\hline Triglycerides $\mathrm{mmol} \cdot \mathrm{L}^{-1}$ & $1.4(1.0-2.2)$ & $1.4(0.8-1.9)$ & $1.5(1.1-1.5)$ \\
\hline Ankle-brachial index ratio & $0.55(0.49-0.70)$ & $0.58(0.51-0.58)$ & $0.53(0.45-0.53)$ \\
\hline PAD history $\geqslant 4$ years & 31 (37) & $16(36)$ & 15 (39) \\
\hline Critical ischaemia & 11 (13) & $6(13)$ & $5(13)$ \\
\hline
\end{tabular}

Data are presented as $n$, mean \pm SD, $n(\%)$ or median (interquartile range). AHI: apnoea/hypopnoea index; $\mathrm{BMI}$ : body mass index; LVEF: left ventricular ejection fraction; LDL: low-density lipoprotein; HDL/cholesterol: highdensity lipoprotein/total cholesterol ratio; PAD: peripheral arterial disease. ${ }^{\#}$ : comparisons between the two groups were performed with the Student's t-test (normal distribution) or the Mann-Whitney U-test (skewed distribution) for continuous variables and the exact Fisher's exact test for categorical variables; " $p<0.0001 .{ }^{*}: p<0.05$. 
TABLE 2 Long-term medication use according to severity of obstructive sleep apnoea

\begin{tabular}{lccc} 
& All patients & AHI $<\mathbf{2 0}$ events $\cdot \mathbf{h}^{-\mathbf{1}}$ & AHI $\geqslant \mathbf{2 0}$ events $\cdot \mathbf{h}^{-\mathbf{1}}$ \\
\hline Subjects & 84 & 45 & 39 \\
Any antihypertensive & $76(91)$ & $39(87)$ & $37(95)$ \\
Beta-blocker & $53(63)$ & $25(56)$ & $28(72)$ \\
ACE inhibitor or ATR blocker & $50(60)$ & $27(60)$ & $23(59)$ \\
Three or more antihypertensives & $19(23)$ & $9(20)$ & $10(26)$ \\
Statins & $52(62)$ & $28(62)$ & $24(62)$ \\
Acetylsalicylic acid & $70(83)$ & $38(84)$ & $32(82)$ \\
Clopidogrel & $22(26)$ & $14(31)$ & $8(21)$ \\
Warfarin & $6(7)$ & $4(9)$ & $2(5)$ \\
Opiates & $21(25)$ & $9(20)$ & $12(31)$ \\
Benzodiazepines & $13(16)$ & $8(18)$ & $5(13)$ \\
\hline
\end{tabular}

Data are presented as $\mathrm{n}$ or $\mathrm{n}(\%)$. None of the comparisons between the groups, performed with Fisher's exact test, indicated significant difference. AHI: apnoea/hypopnoea index; ACE: angiotensin-converting enzyme; ATR: angiotensin receptor.

10-20 events $\cdot \mathrm{h}^{-1}$ appeared to be nonsignificant (HR 0.3 (95\% CI 0.06-1.9); $\mathrm{p}=0.2$ ). Therefore, the patient groups were combined for the final AHI threshold of $\geqslant 20$ events $\cdot h^{-1}$. ODI was excluded from Cox regression analysis due to its high correlation with the AHI $(r=0.92)$ to avoid the multicollinearity problem in the multivariate model. The results of the univariate and multivariate Cox regression analyses with the AHI $\geqslant 20$ events $\cdot \mathrm{h}^{-1}$ as the threshold for all significant variables are summarised in table 5. In the comparison between patients with and without MACCE, the AHI was significantly higher in patients suffering from MACCE ( $\mathrm{p}=0.049$ with Mann-Whitney U-test). In the univariate Cox regression, fatal MACCE alone were significantly more common in patients with an AHI $\geqslant 20$ events $\cdot h^{-1}$ (HR 5.8 (95\% CI 1.3-27.2); $\mathrm{p}=0.02$ ). Other significant predictors associated with MACCE (fatal or non-fatal) were age $(p=0.03)$, coronary artery disease $(p=0.004)$, a history of PAD for $\geqslant 4$ years $(\mathrm{p}=0.04)$, the nadir of nocturnal oxygen desaturation $(\mathrm{p}=0.03)$ and HDL/total cholesterol ratio $(\mathrm{p}=0.02)$. In the multivariate Cox regression analysis, OSA with an AHI $\geqslant 20$ events $\cdot \mathrm{h}^{-1}$ (HR $5.1(95 \%$ CI 1.9-13.9); $\mathrm{p}=0.001$ ) and a previous diagnosis of coronary artery disease (HR 4.4 (95\% CI 1.8-10.6); $\mathrm{p}=0.001)$ remained the strongest independent predictors. The risk of MACCE was also increased by a PAD history of $\geqslant 4$ years ( $\mathrm{HR} 3.8$ (95\% CI 1.3-11.5); $\mathrm{p}=0.02$ ) and a deteriorating HDL/total cholesterol ratio (HR 0.95 per $1 \%$ change (95\% CI 0.90-1.00); p=0.048). A Kaplan-Meier plot for MACCE-free survival according to the AHI $\geqslant 20$ events $\cdot \mathrm{h}^{-1}$ threshold is shown in figure 2 .

TABLE 3 Sleep variables according to severity of obstructive sleep apnoea

\begin{tabular}{|c|c|c|c|}
\hline & All patients & $\begin{array}{c}\mathrm{AHI}^{\#} \\
<20 \text { events } \cdot \mathrm{h}^{-1}\end{array}$ & $\begin{array}{c}\mathrm{AHI}^{\#} \\
\geqslant 20 \text { events } \cdot \mathrm{h}^{-1}\end{array}$ \\
\hline Subjects & 84 & 45 & 39 \\
\hline$A H I$ events $\cdot h^{-1}$ & $18(9-35)$ & $10(4-14)$ & $36(25-48)^{\pi}$ \\
\hline ODI events $\cdot h^{-1}$ & $17(8-34)$ & $9(4-14)$ & $35(25-50)^{\oplus}$ \\
\hline CSA \% & $22(14-27)$ & $21(0-28)$ & $23(18-27)$ \\
\hline Berlin Questionnaire score 2-3 & $37(46)$ & $17(38)$ & $20(56)$ \\
\hline ESS & $4(3-8)$ & $4(3-8)$ & $5(3-8)$ \\
\hline Excessive daytime sleepiness (ESS >10) & $8(10)$ & $7(16)$ & $1(3)$ \\
\hline Arousal index events $\cdot h^{-1}$ & $20(13-29)$ & $15(10-22)$ & $23(17-31)^{* * *}$ \\
\hline $\mathrm{SaO}_{2}$ nadir $\%$ & $85(80-88)$ & $86(83-89)$ & $81(78-86)^{* * *}$ \\
\hline $\mathrm{SaO}_{2}$ mean $\%$ & 94 (92-95) & 94 (92-95) & $94(92-95)$ \\
\hline $\mathrm{SaO}_{2} \mathrm{~T} 90 \mathrm{~min}$ & $4(1-21)$ & $1(0-10)$ & $7(2-51) * *$ \\
\hline
\end{tabular}

Data are presented as $\mathrm{n}$, median (interquartile range) or $\mathrm{n}$ (\%). AHI: apnoea/hypopnoea index; ODI: oxygen desaturation index; CSA: central sleep apnoea; ESS: Epworth Sleepiness Scale; $\mathrm{SaO}_{2}$ : arterial oxygen saturation; T90: time below $90 \%$ saturation. ${ }^{\#}$ : comparisons between the two groups were performed using the Mann-Whitney U-test for continuous variables and the Fisher's exact test for categorical variables; ": $p<0.0001$. $^{* *}: p<0.01 ;{ }^{* * *}: p<0.001$. 
TABLE 4 Major end-points according to severity of obstructive sleep apnoea

\begin{tabular}{lccc} 
& All patients & $\begin{array}{c}\text { AHI } \\
<\mathbf{2 0} \text { events } \mathbf{h}^{-\mathbf{1}}\end{array}$ & $\begin{array}{c}\text { AHI } \\
\geqslant \mathbf{2 0} \text { events } \cdot \mathbf{h}^{-\mathbf{1}}\end{array}$ \\
\hline Subjects & & 45 & 39 \\
All-cause mortality & $18(21)$ & $6(13)$ & $12(31)$ \\
Combined MACCE & $23(27)$ & $6(13)$ & $17(44)^{* *}$ \\
Fatal MACCE & $11(13)$ & $2(4)$ & $9(23)^{*}$ \\
$\quad$ Cardiac death & $9(11)$ & $1(2)$ & $8(18)^{*}$ \\
$\quad$ Stroke & $2(2)$ & $1(2)$ & $1(2)$ \\
Non-fatal AMI & $7(8)$ & $2(4)$ & $5(13)$ \\
Coronary revascularisation & $4(5)$ & $2(4)$ & $2(5)$ \\
Unstable angina pectoris (hospitalisation) & $1(1)$ & $0(0)$ & $1(3)$ \\
Limb complication & $22(26)$ & $10(22)$ & $0(31)$ \\
$\quad$ Amputation & $3(4)$ & $10(0)$ & $3(8)$ \\
Assisted patency (PTA included) & $19(23)$ & $10(22)$ & $9(23)$ \\
\hline
\end{tabular}

Data are presented as $\mathrm{n}$ or $\mathrm{n}(\%)$. AHI: apnoea/hypopnoea index; MACCE: major adverse cardiovascular or cerebrovascular event; AMI: acute myocardial infarction; PTA: percutaneous transluminal angioplasty. ${ }^{*}: \mathrm{p}<0.05$; $^{* *}: \mathrm{p}<0.01$, calculated using univariate Cox regression analysis.

\section{Discussion}

The main result of this study is that occult OSA with an AHI of $\geqslant 20$ events $\cdot \mathrm{h}^{-1}$ is associated with major long-term cardiovascular and cerebrovascular events in patients with severe PAD undergoing peripheral surgical revascularisation. Pre-existing coronary artery disease was another independent predictor of poor prognosis whereas other established risk factors, such as hypertension and diabetes, were not significant.

Although OSA, along with coronary artery disease, was the best independent predictor of MACCE in this study, the major events were also associated with decreased HDL/total cholesterol ratio. This is in line with previous data indicating that impaired lipid metabolism is a principal risk factor for cardiovascular morbidity and mortality. We have previously demonstrated that OSA in our study sample was also linked to decreased HDL/total cholesterol ratio [17]. Intermittent hypoxia, caused by the repeated breathing cessations in OSA, is known to promote sympathetic activation, oxidative stress, systemic inflammation and endothelial dysfunction $[6,10,11]$. Importantly, a recent study proved a causal relationship between minimally symptomatic OSA and endothelial dysfunction [28]. Therefore, OSA is associated with several common denominators that are also the key mechanisms in the pathophysiology of atherosclerosis. Furthermore, the repeated apnoeas per se may directly predispose to myocardial infarction through a variety of mechanisms (e.g. left ventricular hypertrophy, systolic and diastolic dysfunction, reduced supply and increased demand for oxygen in the myocardium) [6]. In this study, we also showed that a longer PAD history ( $\geqslant 4$ years) was associated with increased morbidity and mortality. Accordingly, the current results further support the view that OSA has a close relationship with endothelial dysfunction and the pathophysiology of atherosclerosis.

TABLE 5 Univariate and multivariate predictors of major adverse cardiovascular or cerebrovascular events

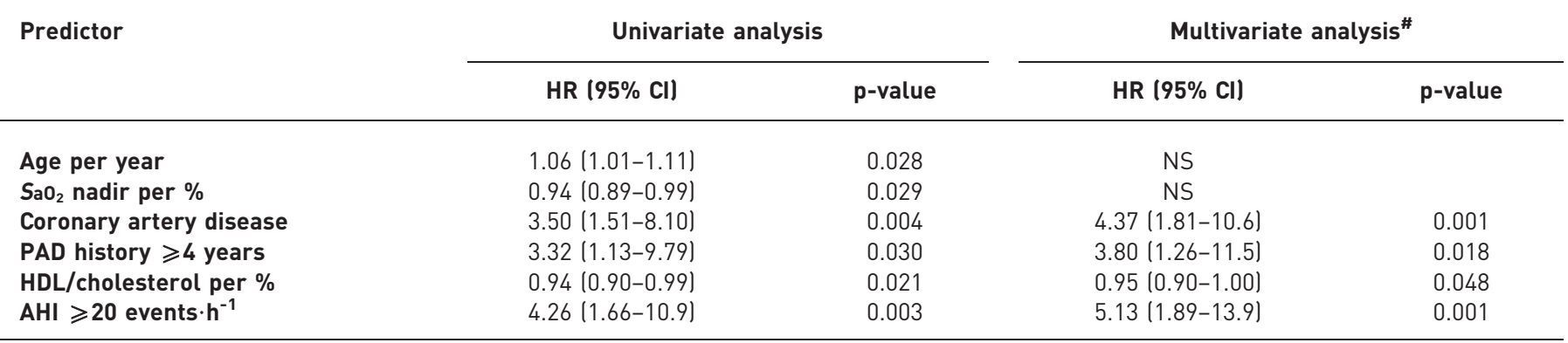

HR: hazard ratio; $\mathrm{SaO}_{2}$ : arterial oxygen saturation; PAD: peripheral arterial disease; HDL/cholesterol: high-density lipoprotein/total cholesterol ratio; $\mathrm{AHI}$ : apnoea/hypopnoea index; NS: not selected into the final model. \#: performed using stepwise selection method (inclusion criteria $\mathrm{p}<0.05$ and exclusion criteria $p \geqslant 0.05)$. 


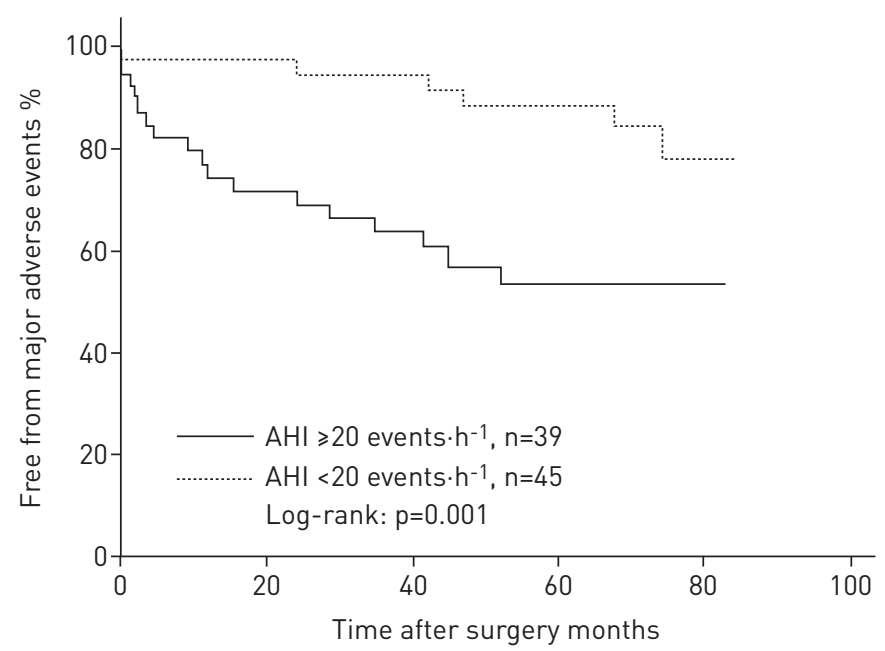

FIGURE 2 Kaplan-Meier survival plot for patients free from major adverse cardiovascular and cerebrovascular events according to the apnoea/hypopnoea index (AHI) threshold of $\geqslant 20$ events $\cdot \mathrm{h}^{-1}$. No cases were censored.

An elevated cardiac troponin level within three post-operative days after major vascular surgery is well documented to be a strong independent predictor for mortality within the first post-operative year and to a lesser extent for the longer term [2]. According to earlier studies, up to one-third of standard PAD patients after peripheral bypass should have a cardiac troponin $\mathrm{T}$ release $>0.03 \mu \mathrm{g} \cdot \mathrm{L}^{-1}[2,29]$. However, that applied in only one of the current patients demonstrating a relatively low cardiac risk compared to standard surgical PAD population. One very plausible explanation is that current patients were somewhat younger and healthier than the standard surgical PAD population. In addition, patients with atrial fibrillation and congestive heart failure, who are likely to have more severe systemic atherosclerosis and higher cardiac risk, were not enrolled in this study. Therefore, due to strict exclusion criteria including all OSA syndrome patients, this study lacks some of the major well-established cardiac risk factors, further addressing the clinical importance of occult OSA in these high-risk PAD patients. Current results suggest that significant OSA in PAD patients needing surgical revascularisation is independently associated with long-term mortality and to an even greater extent in the presence of coronary artery disease.

The American Society of Anesthesiologists Task Force advocates systematic screening for OSA and treatment with continuous positive airway pressure (CPAP) as preoperative management of patients with severe OSA [30]. In this study, we were unable to show a relationship between OSA and perioperative major adverse events in patients with $\mathrm{PAD}$, although they are considered as one of the most high-risk groups of surgical patients $[2,3]$. In addition to small sample size, this is probably, at least in part, due to the exclusion criteria as discussed previously. Before a large-scale screening for OSA in surgical PAD patients can be justified, an efficient treatment intervention improving the outcome of these patients should be investigated. Mounting evidence shows that CPAP prevents fatal and non-fatal cardiovascular events in patients with severe OSA [16, 27, 31, 32]. However, the efficacy of CPAP treatment in surgical PAD patients with OSA remains to be established. Also, a more aggressive pharmacological secondary prevention, even without CPAP treatment, may be beneficial in these patients. According to the current results, patients with coronary artery disease, a PAD history of several years and impaired lipid metabolism may be a feasible group for screening and intervention.

There are important limitations to be considered. The current observational study design does not allow any conclusions on causal relationships. Our study sample and number of events was small, partly due to rigorous patient selection. However, this can be considered an advantage since we were able to show the effect of OSA on outcome with fewer confounding factors. In line with previous literature, we used an AHI $\geqslant 20$ events $\cdot \mathrm{h}^{-1}$ as a cut-off level, which corresponds closely to the mean and median AHI observed in this study $[16,27]$. However, we were unable to demonstrate a continuous trend between the increase in AHI and the risk of MACCE. This supports an earlier large cohort study showing that the relationship between the AHI and cardiovascular risk is not linear [33]. Half of the screened patients were excluded according to protocol criteria and were not evaluated for the presence of OSA or any symptoms related to it. This may have resulted in disproportionate representation of minimally symptomatic and asymptomatic patients. Therefore, we cannot draw any conclusions regarding the prevalence of OSA-related sleepiness in standard PAD patients needing surgical revascularisation. Instead, this study shows that PAD patients with an AHI 
$\geqslant 20$ events $\cdot \mathrm{h}^{-1}$ at the time of peripheral arterial bypass surgery have an increased risk for long-term serious cardiac events and cardiac death, even without OSA-related sleepiness.

In conclusion, significant OSA (AHI $\geqslant 20$ events $\cdot \mathrm{h}^{-1}$ ) is associated with long-term morbidity and mortality in PAD patients needing peripheral surgical revascularisation. In addition, further studies are warranted to find out whether CPAP treatment is able to bring any clinical benefit to these patients.

\section{Acknowledgements}

We would like to thank N. Karppinen (Sleep Research Unit, University of Turku, Turku, Finland) and K. Leivo (Division of Perioperative Services, Intensive Care Medicine and Pain Management, Turku University Hospital, Turku, Finland) for technical support.

\section{References}

1 Hirsch AT, Criqui MH, Treat-Jacobson D, et al. Peripheral arterial disease detection, awareness and treatment in primary care. JAMA 2001; 286: 1317-1324.

2 Landesberg G, Shatz V, Akopnik I, et al. Association of cardiac troponin, CK-MB, and postoperative myocardial ischemia with long-term survival after major vascular surgery. J Am Coll Cardiol 2003; 42: 1547-1554.

3 Eagle KA, Berger PB, Calkins H, et al. ACC/AHA guideline update for perioperative cardiovascular evaluation for noncardiac surgery. Circulation 2002; 105: 1257-1267.

4 Halm EA, Browner WS, Tubau JF, et al. Echocardiography for assessing cardiac risk in patients having noncardiac surgery: the DECREASE-V Pilot Study. Ann Intern Med 1996; 125: 433-441.

5 Poldermans D, Schouten O, Vidakovic R, et al. A clinical randomized trial to evaluate the safety of a noninvasive approach in high-risk patients undergoing major vascular surgery. J Am Coll Cardiol 2007; 49: 1763-1769.

6 Bradley TD, Floras JS. Obstructive sleep apnoea and its cardiovascular consequences. Lancet 2009; 373: 82-93.

7 Kendzerska T, Mollayeva T, Gershon AS, et al. Untreated obstructive sleep apnea and the risk for serious long-term adverse outcomes: a systematic review. Sleep Med Rev 2014; 1: 49-59.

8 Peppard PE, Young T, Palta M, et al. Prospective study of the association between sleep-disordered breathing and hypertension. N Engl J Med 2000; 342: 1378-1384.

9 Ip MS, Lam B, Ng MM, et al. Obstructive sleep apnea is independently associated with insulin resistance. Am J Respir Crit Care Med 2002; 165: 670-676.

10 Kohler M, Craig S, Nicoll D, et al. Endothelial function and arterial stiffness in minimally symptomatic sleep apnea. Am J Respir Crit Care Med 2008; 178: 984-988.

11 Jelic S, Padeletti M, Kawut SM, et al. Inflammation, oxidative stress and repair capacity of the vascular endothelium in obstructive sleep apnea. Circulation 2008; 117: 2270-2278.

12 Drager LF, Bortolotto LA, Maki-Nunes C, et al. The incremental role of obstructive sleep apnoea on markers of atherosclerosis in patients with metabolic syndrome. Atherosclerosis 2010; 208: 490-495.

13 Mason RH, Ruegg G, Perkins J, et al. Obstructive sleep apnea in patients with abdominal aneurysms: highly prevalent and associated with aneurysm expansion. Am J Respir Crit Care Med 2011; 183: 668-674.

14 Mooe T, Franklin KA, Holmstrøm K, et al. Sleep-disordered breathing and coronary artery disease: long-term prognosis. Am J Respir Crit Med 2001; 164: 1910-1913.

15 Yaggi HK, Concato J, Kernan WN, et al. Obstructive sleep apnea as a risk factor for stroke and death. N Engl J Med 2005; 353: 2034-2041.

16 Gooneratne NS, Richards KC, Joffe M, et al. Sleep-disordered breathing with excessive daytime sleepiness is a risk factor for mortality in older adults. Sleep 2011; 34: 435-442.

17 Utriainen KT, Airaksinen JK, Polo O, et al. Unrecognised obstructive sleep apnoea is common in severe peripheral arterial disease. Eur Respir J 2013; 41: 616-620.

18 American Diabetes Association. Diagnosis and classification of diabetes mellitus. Diabetes Care 2008; 31: Suppl. 1, S55-S60.

19 Grundy SM, Cleeman JI, Daniels SR, et al. Diagnosis and management of the metabolic syndrome: an American Heart Association/National Heart, Lung and Blood Institute scientific statement. Circulation 2005; 112: 2735-2752.

20 Sleep-related breathing disorders in adults: recommendations for syndrome definition and measurement techniques in clinical research. The report of an American Academy of Sleep Medicine Task Force. Sleep 1999; 22: 667-689.

21 Rechtschaffen A, Kales A, eds. A Manual of Standardization Terminology: Techniques and Scoring Systems for Sleep Stages of Human Subjects. Los Angeles, Brain Information Services/Brain Research Institute, University of California at Los Angeles, 1968.

22 Argod J, Pépin JL, Lévy P. Differentiating obstructive and central sleep respiratory events through pulse transit time. Am J Respir Crit Care Med 1998; 158: 1778-1783.

23 Argod J, Pépin JL, Smith RP, et al. Comparison of esophageal pressure with pulse transit time as a measure of respiratory effort for scoring obstructive nonapneic respiratory events. Am J Respir Crit Care Med 2000; 162: 87-93.

24 Johns MW. A new method for measuring daytime sleepiness: the Epworth sleepiness scale. Sleep 1991; 14: 540-545.

25 Netzer NC, Stoohs RA, Netzer CM, et al. Using the Berlin Questionnaire to identify patients at risk for the sleep apnea syndrome. Ann Intern Med 1999; 131: 485-491.

26 Thygesen K, Alpert JS, Jaffe AS, et al. Third universal definition of myocardial infarction. J Am Coll Cardiol 2012; 60: 1581-1598.

27 Martínez-García MA, Soler-Cataluña JJ, Ejarque-Martínez L, et al. Continuous positive airway pressure treatment reduces mortality in patients with ischemic stroke and obstructive sleep apnea: a 5-year follow-up study. Am J Respir Crit Care Med 2009; 180: 36-41.

28 Kohler M, Craig S, Pepperell JC, et al. CPAP improves endothelial function in patients with minimally symptomatic OSA: results from a subset study of the MOSAIC trial. Chest 2013; 144: 896-902. 
29 Levy M, Heels-Ansdell D, Hiralal R, et al. Prognostic value of troponin and creatine kinase muscle and brain isoenzyme measurement after noncardiac surgery: a systematic review and meta-analysis. Anesthesiology 2011; 114: 796-806.

30 Gross JB, Bachenberg KL, Benumof JL, et al. Practice guidelines for the perioperative management of patients with obstructive sleep apnea: a report by the American Society of Anesthesiologists Task Force on perioperative management of patients with obstructive sleep apnea. Anesthesiology 2006; 104: 1081-1093.

31 Campos-Rodriguez F, Martinez-Garcia MA, de la Cruz-Moron I, et al. Cardiovascular mortality in women with obstructive sleep apnea with or without continuous positive airway pressure treatment: a cohort study. Ann Intern Med 2012; 156: 115-122.

32 Marin JM, Carrizo SJ, Vicente E, et al. Long-term cardiovascular outcomes in men with obstructive sleep apnoeahypopnoea with or without treatment with continuous positive airway pressure: an observational study. Lancet 2005; 365: 1046-1053.

33 Shahar E, Whitney CW, Redline S, et al. Sleep-disordered breathing and cardiovascular disease: cross-sectional results of the Sleep Heart Health Study. Am J Respir Crit Care Med 2001; 163: 19-25. 